\title{
High-precision structural analysis of subnuclear complexes in fixed and live cells via spatially modulated illumination (SMI) microscopy
}

\author{
Jürgen Reymann ${ }^{1}$, David Baddeley ${ }^{1}$, Manuel Gunkel ${ }^{1}$, Paul Lemmer ${ }^{1}$, Werner Stadter ${ }^{2}$, Thibaud Jegou ${ }^{3}$, \\ Karsten Rippe $^{3}$, Christoph Cremer ${ }^{1,4}$ \& Udo Birk ${ }^{1,5 *}$ \\ ${ }^{1}$ Applied Optics and Information Processing, Kirchhoff Institute of Physics, University of Heidelberg, INF 227, \\ 69120, Heidelberg, Germany; Tel: +49-622-1549274; Fax: +49-622-1549112; E-mail: udo.birk@kip.uni- \\ heidelberg.de; ${ }^{2}$ University of Applied Sciences, Hamburg, Germany; ${ }^{3}$ Research Group Genome Organization \\ and Function, German Cancer Research Centre (DKFZ) \& Bioquant Centre, University of Heidelberg, \\ Heidelberg, Germany; ${ }^{4}$ Institute for Pharmacy and Molecular Biotechnology (IPMB)/Bioquant Centre, \\ University of Heidelberg, Germany \& Institute for Molecular Biophysics, The Jackson Laboratory, Bar Harbor, \\ USA; ${ }^{5}$ In-vivo Optical Imaging Group, Institute of Electronic Structure and Laser, FORTH, Heraklion, Greece \\ * Correspondence
}

Key words: FPALM, live cell imaging, localization microscopy, nanosizing, PALM, PALMIRA, SALM, SMI microscopy, SPDM, STORM, Vertico-SMI microscope

\begin{abstract}
Spatially modulated illumination (SMI) microscopy is a method of wide field fluorescence microscopy featuring interferometric illumination, which delivers structural information about nanoscale architecture in fluorescently labelled cells. The first prototype of the SMI microscope proved its applicability to a wide range of biological questions. For the SMI live cell imaging this system was enhanced in terms of the development of a completely new upright configuration. This so called Vertico-SMI transfers the advantages of SMI nanoscaling to vital biological systems, and is shown to work consistently at different temperatures using both oil- and waterimmersion objective lenses. Furthermore, we increased the speed of data acquisition to minimize errors in the detection signal resulting from cellular or object movement. By performing accurate characterization, the present Vertico-SMI now offers a fully-fledged microscope enabling a complete three-dimensional (3D) SMI data stack to be acquired in less than 2 seconds. We have performed live cell measurements of a tet-operator repeat insert in U2OS cells, which provided the first in vivo signatures of subnuclear complexes. Furthermore, we have successfully implemented an optional optical configuration allowing the generation of high-resolution localization microscopy images of a nuclear pore complex distribution.
\end{abstract}

$\begin{array}{ll}\text { Abbreviations } \\ \text { AHF } & \begin{array}{l}\text { Analysentechnik AG, Tübingen, Germany } \\ \text { axial intensity distribution }\end{array} \\ \text { AID } & \text { Beam splitter } \\ \text { BS } & \text { confocal laser scanning microscopy } \\ \text { CLSM } & \text { dichroic mirrors } \\ \text { DM } & \text { data acquisition } \\ \text { DAQ } & \text { fluorescence resonance energy transfer } \\ \text { FRET } & \text { full width at half maximum }\end{array}$

$\begin{array}{ll}\text { GUI } & \text { graphical user interface } \\ \text { LED } & \text { light-emitting diode } \\ \text { OL } & \begin{array}{l}\text { oil-immersion objective lens } \\ \text { OTF }\end{array} \\ \text { optical transfer function } \\ \text { PSF } & \text { point spread function } \\ \text { PALM } & \text { photoactivated localization microscopy } \\ \text { PALMIRA } & \text { PALM with independently running acquisition } \\ \text { RT } & \text { room temperature } \\ \text { SD } & \text { standard deviation } \\ \text { SNR } & \text { signal-to-noise ratio }\end{array}$


SMI spatially modulated illumination

SPDM spectral precision distance microscopy / spectral position determination microscopy

SALM spectrally assigned localization microscopy

STED stimulated emission depletion

STORM sub-diffraction-limited imaging by stochastic optical reconstruction

\section{Introduction}

Spatially modulated illumination (SMI) microscopy is a method of wide-field fluorescence microscopy using structured illumination to obtain additional information about sizes and relative positions of fluorescently marked target regions, thus allowing also high-precision measurements of the distances between them. To generate the illumination pattern, two counterpropagating laser beams are brought to constructive interference, establishing a standing-wave field (Lanni et al. 1986, Bailey et al. 1993). In contrast to focused laser light techniques (Hell et al. 1994, Klar et al. 2000, Dyba \& Hell 2002, Egner et al. 2002) or structured illumination (Gustafsson et al. 1995, 1996, Frohn et al. 2000, 2001, Heintzmann et al. 2002), the optical resolution itself is not enhanced. Nevertheless, in combination with high-precision axial positioning this technique of far-field light microscopy allows the nondestructive analysis of complex spatial arrangements (Albrecht et al. 2002) inside relatively thick transparent specimens such as the cell nucleus and enables size measurements at molecular dimensions of a few tens of nanometers (Failla et al. 2002b, 2003).

SMI microscopy is now an established method for the analysis of topological arrangements of mammalian genome structure. In combination with novel approaches for fluorescence labelling, the SMI 'nanosizing' technique has proved its applicability for a wide range of biological questions when using fixed cell preparations (Martin et al. 2004, Hildenbrand et al. 2005, Mathée et al. 2006). Nevertheless, the samples that have been analysed so far were fixed specimens and the influence of fixation procedures on the overall cell structure and in particular on the nanostructure of the genome has not yet been clarified (Hendzel \& Bazett-Jones 1997, Kozubek et al. 2000). For instance, it is still not known exactly how different fixation methods influence these features.
For many biological questions it is of utmost interest to image the pure, uninfluenced genome structure as well as the dynamical behaviour of subnuclear complexes and physiological processes. We have thus enhanced the SMI principle to allow live-cell imaging for high-precision structural analysis below the conventional optical resolution limit.

The Vertico-SMI was developed as an upright configuration of the SMI microscope and we present this new set-up as well as the physical characteristics of the whole system. As a key aspect for the analysis of living biological specimens, the speed of data acquisition had to be increased in order to minimize influences resulting from dynamic processes, while still being able to extract useful signals for size determination. We show the reliability of Vertico-SMI measurements even under high data acquisition rates (presently $1.8 \mathrm{~s}$ for a complete 3D SMI data stack) and the first in vivo signatures of subnuclear loci in living cells. Furthermore, the Vertico-SMI set-up allows switching between two optical configurations in order to facilitate both SMI and localization microscopy using photoconvertible molecular markers.

\section{SMI microscopy}

For the analysis of 3D nanostructures SMI microscopy applies methods of point spread function (PSF) engineering, enabling the quantitative characterization of the sizes of fluorescent objects in a range of about 30-200 $\mathrm{nm}$ in axial direction. Using structured wide-field illumination in combination with widefield detection, this technique provides additional spatial information about the analysed objects compared with conventional microscopy techniques such as confocal laser scanning microscopy (CLSM).

\section{Optical layout}

The experimental set-up of the first prototype of the SMI microscope, placed horizontally at an optical table, has evolved during time and in the following the current configuration is described (see Figure 1). For illumination, three laser sources are available for excitation at $\lambda_{\text {exc }}=488 \mathrm{~nm}, 568 \mathrm{~nm}$ and $647 \mathrm{~nm}$ (Lexel 95-4, Lexel 95L-K and Lexel 95-K, Lexel Laser, Fremont, CA, USA), which additionally can be independently switched with shutters before being 


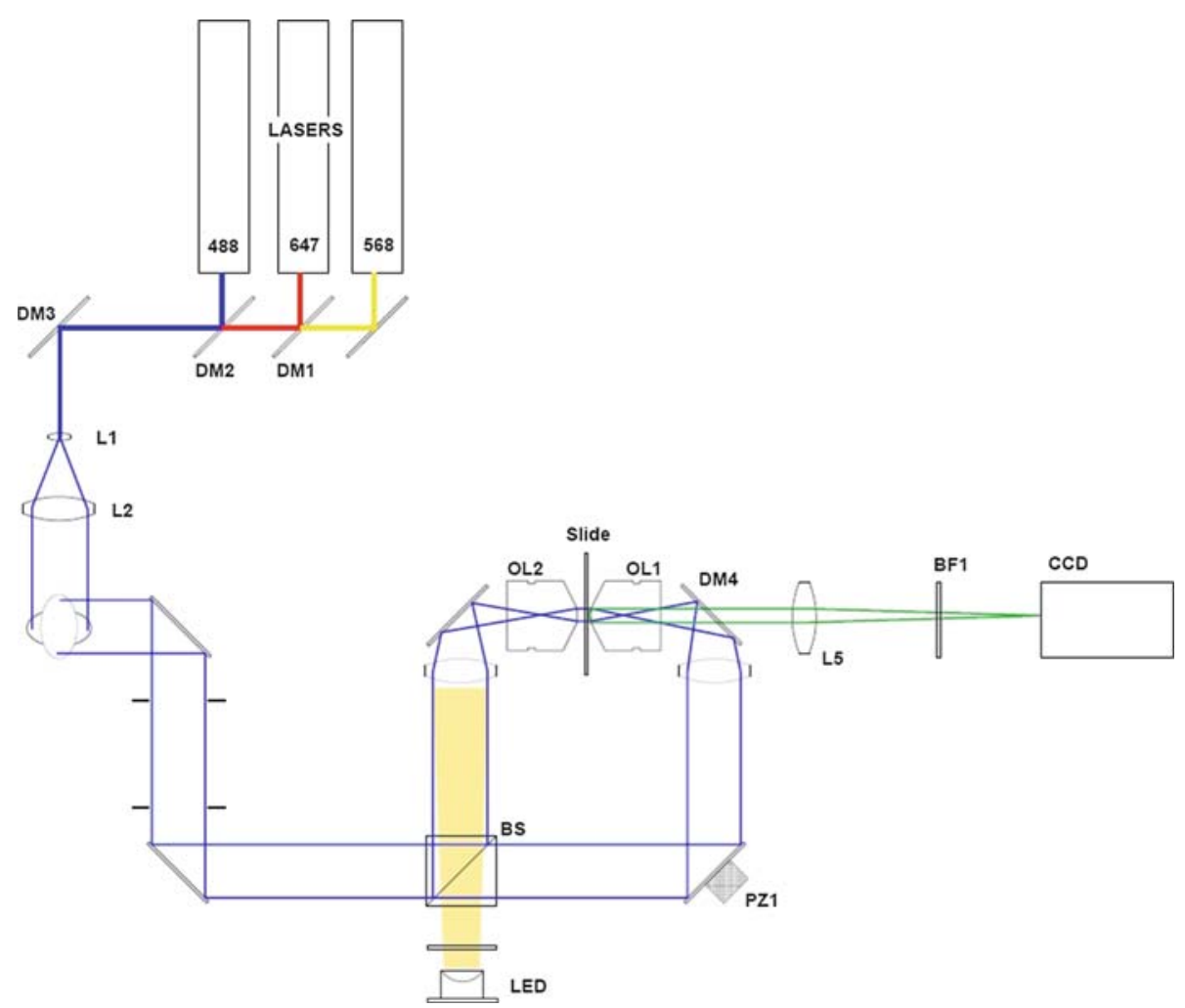

Figure 1. Current experimental set-up of the first prototype of the SMI microscope (top view). For a complete description of the individual components see text.

combined with the dichroic mirrors DM1 and DM2 (AHF: Analysentechnik AG, Tübingen, Germany). The dichroic DM3 works as a clean-up filter and reflects the three laser lines into the collimator, consisting of two achromates (L1 and L2) (Linos Photonics, Göttingen, Germany) with $10 \mathrm{~mm}$ and $100 \mathrm{~mm}$ focal length respectively, to expand the beam to a diameter of approximately $20 \mathrm{~mm}$. The expanded laser beam is then conveyed to the breadboard (an additional breadboard made from super-invar on the optical table is used for the interferometric unit in order to guarantee maximal thermal stability) via a periscope, where it is split by a 50:50 beam splitter (BS) (Edmund Optics, Karlsruhe, Germany), to yield two coherent counterpropagating and collimated laser beams which are focused into the back focal plane of two opposing oil immersion objective lenses (OL1 and OL2) $(100 \times, \mathrm{NA}=1.4$, Leica, Bensheim, Germany). This results in a collimated laser beam leaving each objective lens. Interference between these two beams leads to a standing-wave field in the space between the two objective lenses, and hence a $\cos ^{2}$ shape of the intensity along the optical axis. Samples may be prepared using ordinary object slides (or coverslips or both combined) and are then placed between the two objective lenses and moved along the optical axis with a piezoelectric stage (Physik Instrumente, Karlsruhe, Germany), thus allowing 3D data stacks of the specimens to be obtained. An additional piezoelectric stage (PZ1) allows the relative phase in the two interferometer arms to be varied. The emission light from the fluorescently labelled target regions, collected by the detection objective lens (OL1), is then separated from the excitation light by a dichroic mirror (DM4) (AHF) and focused with the help of a tube lens (L5) $(1.25 \times$, Leica) onto a highly sensitive black-andwhite CCD camera (SensiCam QE, PCO Imaging, Kelheim, Germany) used for detection. In front of the CCD chip, a blocking filter (BF1) in a filter wheel blocks any remaining laser light and, depending on filter selection, out-of-band fluorescence. Moreover, 
a white light-emitting diode (LED) can be used in transmission mode to locate the focal plane in order to reduce bleaching of the dyes.

\section{Data evaluation}

Owing to the collimated beam in the object space, the complete lateral focal plane can be imaged simultaneously. For data acquisition (DAQ), the objects to be analysed have only to be scanned in one dimension (axially), which leads to more rapid DAQ rates in contrast to confocal microscopy techniques. Nevertheless, the step size in the axial direction cannot be chosen arbitrarily but has to fulfil the Nyquist condition. With distances between the intensity maxima of the standing-wave field (wavefront distance; note that the wavefront distance depends on the laser wavelength) of about $180 \mathrm{~nm}$, this is realized in practice by sampling the objects with an axial step size less than $80 \mathrm{~nm}$; typically, a step size of $40 \mathrm{~nm}$ is used (this slight oversampling contributes to the stability of the subsequent data fitting).

The structured illumination leads to a modified SMI PSF; in the following we present a brief overview where only this axial direction is considered since the SMI technique provides additional size information only in that dimension. The fundamental principles of SMI microscopy have been discussed in detail in previous publications (Albrecht et al. 2002, Failla et al. 2002b, 2003, Spöri et al. 2004). The SMI PSF is the product of the detection PSF corresponding to an ordinary wide-field PSF and the illumination PSF, which is given by the $\cos ^{2}$ shape. It is thus characterized by an additional modulation in the axial direction. The envelope of the wide-field PSF can be calculated from the laser excitation wavelength, the refractive index in the object space and the numerical aperture of the detection objective lens. Sampling of an extended, fluorescent object in the axial direction through the standing-wave field results in a modulated SMI axial profile whose form depends on the size of the object (see Figure 2 (left) for a simulated SMI PSF). In the frequency domain $\left(k_{x}\right.$ and $k_{z}$ ) the illumination pattern consists of three delta functions at 0 and \pm the frequency of the interference pattern. The optical transfer function (OTF) is thus composed of three copies of the wide-field OTF at the locations of the delta functions. The two additional copies at $\pm k_{z}$ provide more information about the object (see Figure 2 (right) for a simulated SMI OTF).

By plotting the detected fluorescence intensity over the object's $z$-position, we obtain the axial intensity distribution (AID), as shown in Figure 3 (simulated AID of an object with a diameter of $100 \mathrm{~nm}$ ). If the focal plane matches an excitation maximum of the standing wave field, the relationship between the acquired AID from a fluorescent object and its size in axial direction is then given by the modulation depth $r=\left(I_{\max }-I_{\min }\right) /\left(I_{\max }\right)=1-\left(A_{1} /\right.$ $\left.A_{2}\right)$, i.e. the ratio between the intensity maxima of the inner $\left(I_{\min }\right)$ and outer $\left(I_{\max }\right)$ envelope of the AID, which is directly correlated to the axial extension of the measured object with a theoretical calibration curve (assuming a given distribution of the fluorophores). The smaller the object the larger is the measured $r$-value.

For data analysis a very comprehensive algorithm for fully automated object detection and size determination was developed and integrated into a MATLAB (The MathWorks, Inc., http://www. mathworks.com) environment which also enables
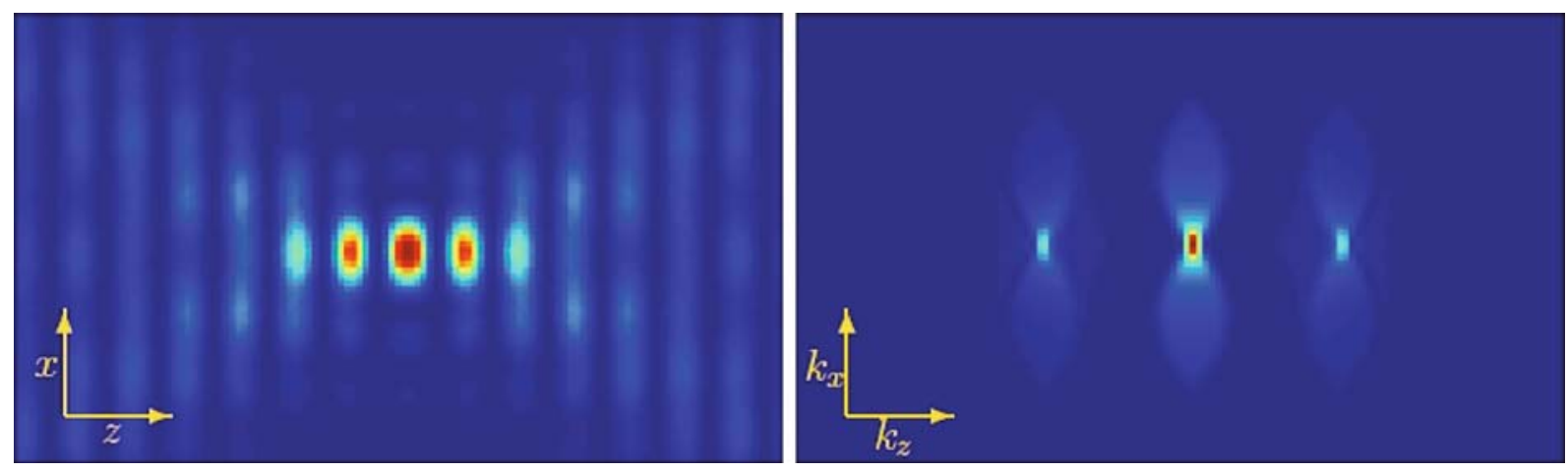

Figure 2. Simulated SMI PSF (left) and OTF (right). 


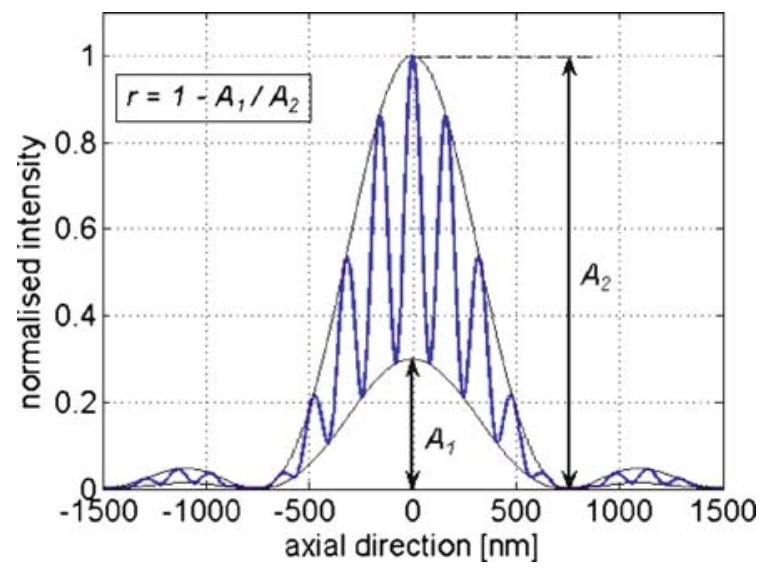

Figure 3. Simulated axial intensity distribution (AID) of a $100 \mathrm{~nm}$ fluorescent microsphere. The axial object size is calculated taking the modulation depth $r$ into account, where $A_{1}$ and $A_{2}$ correspond to the amplitude of the inner and outer envelope respectively. The resulting modulation depth $r$ is then related to the object size with a theoretical calibration curve.

graphical user interface (GUI) interactions. After subtracting the background at the location of each of the automatically detected signals, the algorithm fits the function

$$
I(z)=A \cdot\left[(1-r)+r \cdot \cos ^{2}\left(k\left(z-z_{o}\right)\right)\right] \cdot I_{\text {smooth }}(z)
$$

to an axial profile (AID) extracted for each signal. In this formula $A$ denotes the amplitude, $k$ the wavenumber $\left(2 \pi / \lambda ; \lambda=\lambda_{\text {exc }} / n ; n=\right.$ refraction index $), z$ the axial position of the object slide and $z_{0}$ the displacement of the observed modulation pattern with respect to the origin. The modulation depth is represented by $r$, and $I_{\text {smooth }}(z)$ is the axial profile as it would appear in the absence of the standing-wave field (obtained by applying a low-pass filter to the AID) (Baddeley et al. 2007).

\section{The Vertico-SMI microscope}

In previous efforts, different optical configurations were tested for the first prototype of the SMI microscope in order to establish optimal illumination and detection conditions. At the same time, virtual SMI microscopy (Failla et al. 2002a) was developed to optimize data analysis procedures in order to provide information for better interpretation. Together with the fully automated data analysis tool described above, the SMI microscope has established itself as a fully-fledged and stable microscope for high precision light-optical size measurements of biological samples considerably below the conventional 'Abbe limit'.

Nevertheless, owing to the experimental design of the first prototype of the SMI microscope, the number of biological questions that could be addressed was limited. An advantage of light-optical methods in comparison with high-resolution techniques such as electron microscopy (EM) lies in the possibility of studying 3D structurally intact cells using a large variety of molecular labelling tools. In order to fully access the high potential of lightoptical methods it was essential to enhance our system towards live-cell imaging capability. In addition, this enables the 'real-time' observation of physiological processes and the determination of very precise mobility patterns.

For (long-term) in vivo SMI measurements, several new aspects had to be taken into account. The Vertico-SMI was designed as an upright configuration for horizontal positioning of the object slide in order to integrate water-immersion objective lenses if necessary. This set-up allows the easy implementation of an incubator chamber and flow control systems for keeping the specimens alive. A further decisive aspect resulted in the need for a very stable and compact set-up. The main difference in comparison with the first prototype of the SMI microscope is the possibility to equip the set-up with both oil- and water-immersion objective lenses. Depending on the biological sample and the kind of preparation, the environment of the biological sample will have a different refractive index, requiring appropriately corrected optics. As living cells are usually in aqueous media, the use of water-immersion objective lenses is desirable.

The basic set-up of the Vertico-SMI consists of an aluminium frame which is placed on an optical table and an incubator chamber which encloses the whole set-up (except for the CCD camera, due to the need to cool the CCD chip and the electronics), which enables to maintain an independent atmosphere with constant temperatures up to $50^{\circ} \mathrm{C}$ and with an accuracy of $\sim 0.1^{\circ} \mathrm{C}$.

We specifically calibrated the piezoelectric stage used for the axial object sampling in order to improve DAQ. This led to a more stable and rapid operation in the closed-loop mode, allowing easy conditioning for different operating temperatures. The only restriction concerning a rapid DAQ is consequently imposed by the number of photons 
reaching the CCD chip of the camera per time slice, thus defining a limit given by the integration time.

\section{Object positioning}

For coarse object positioning prior to the actual measurement, translation stages (M-505 ( $x$-axis), M-112 ( $y$-axis) and M-110 (z-axis), Physik Instrumente) with a positioning accuracy down to $50 \mathrm{~nm}$ are used. These units show an absolute positioning stability even under higher temperatures. For axial object sampling during acquisition, the use of a piezoelectric stage with a capacitive control system (P-621, Physik Instrumente) provides a positioning accuracy better than $10 \mathrm{~nm}$ in the closed-loop mode. An additional piezoelectric stage (P-753, Physik Instrumente) placed inside the interferometer enables the adjustment of the wavefront position. The hardware components are controlled via a customwritten software package, which additionally allows computer-controlled heat supply and/or perfusion during live-cell measurements.

\section{Optical layout}

Figure 4 depicts an overview of the optical layout of the Vertico-SMI, which follows the optical set-up of the first prototype of the SMI microscope. The collimated incoming light of an argon ion laser (Spectra-Physics, Newport, Germany) and a krypton ion laser (Lexel 95, Lexel Laser) are operated at wavelengths of $488 \mathrm{~nm}$ and $568 \mathrm{~nm}$, respectively, and with intensities of 50-200 mW. The overlay beam is expanded by a collimator to a beam diameter of about $15 \mathrm{~mm}$, and split by a 50:50 beam splitter (Edmund Optics) into two laser beams of equal intensity. The light is then focused into the back focal plane of the two objective lenses (oil- or waterimmersion, $63 \times, \mathrm{NA}=1.4$ (oil) / 1.2 (water), Leica) through which the standing-wave field is established in the object space. The detection light, collected by the (upper) detection objective lens, is separated from the excitation light with the help of a dichroic mirror (F52-489, AHF) and focused with a $1.0 \times$ tube lens (Leica) onto the CCD chip of a cooled

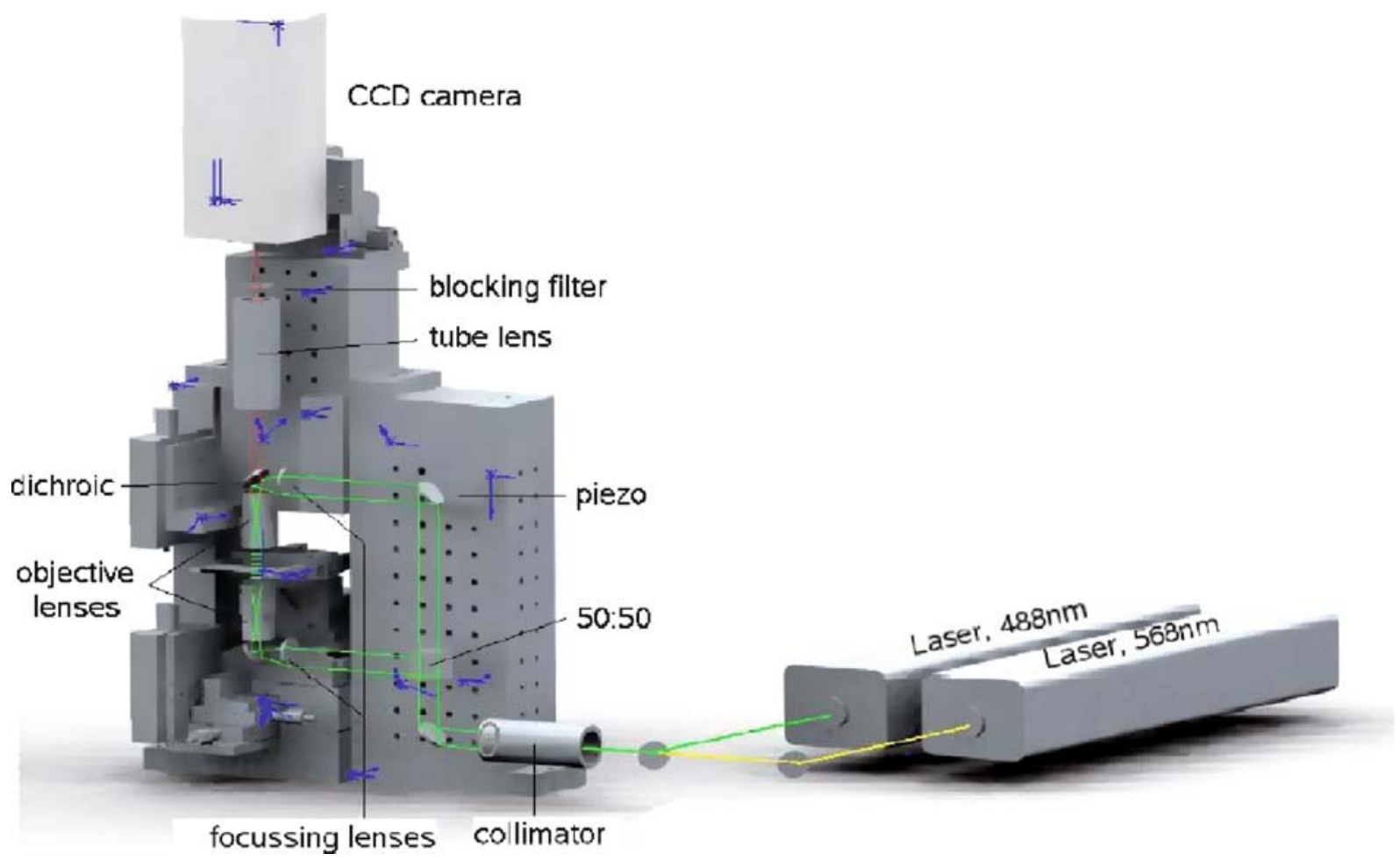

Figure 4. Experimental set-up of the Vertico-SMI microscope. For a description of the individual components see text. 


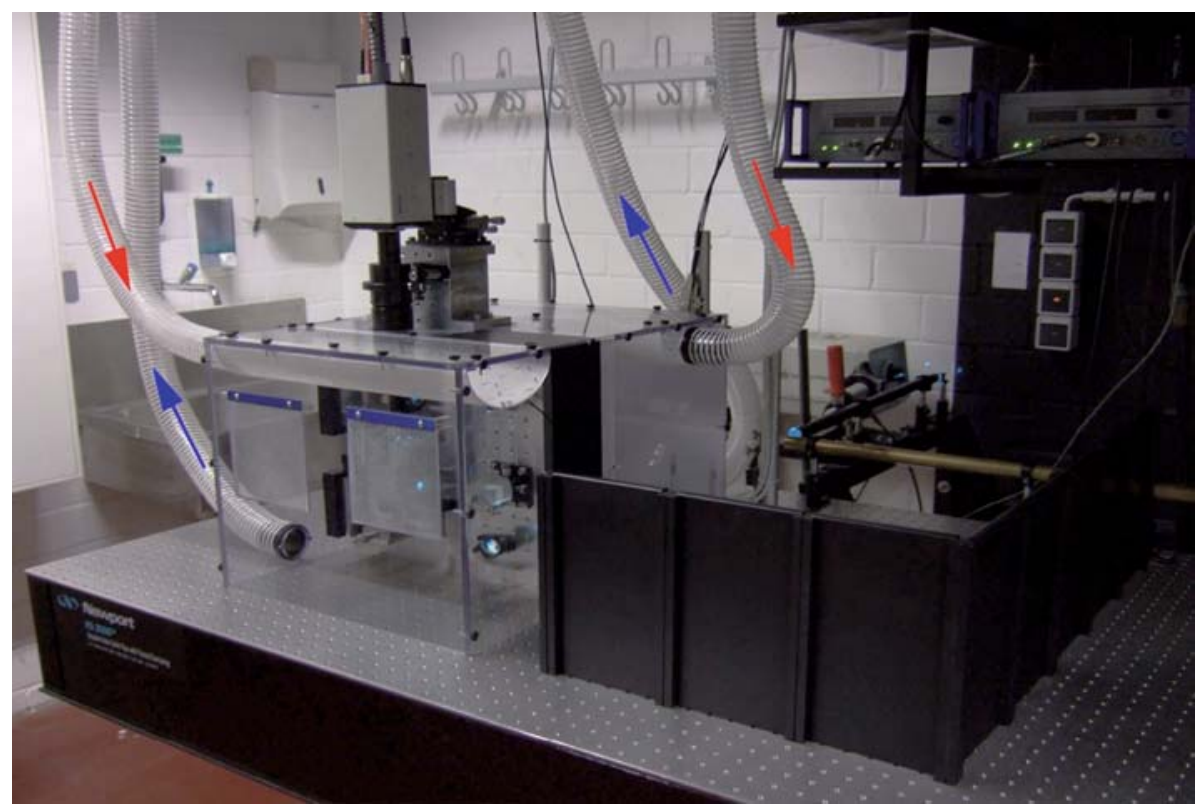

Figure 5. Photograph of the Vertico-SMI with closed incubator chamber. The red arrows (oriented downwards) indicate the supply with heated air and the blue arrows (oriented upwards) the transportation back to the temperature control unit, thus completing the cycle.

black-and-white CCD camera (SensiCam QE, PCO Imaging). An additional blocking filter (F73-491, AHF) in front of the CCD chip blocks residual backreflected laser light. The set-up allows easy switching between different filter sets and emitters for particular applications and excitation wavelengths.

\section{In vivo instrumentation}

We installed an incubator chamber providing stable temperature with a resolution of about $0.1^{\circ} \mathrm{C}$ at the temperature sensor (see Figures 5 and 6). For the establishment of a constant temperature of, say,
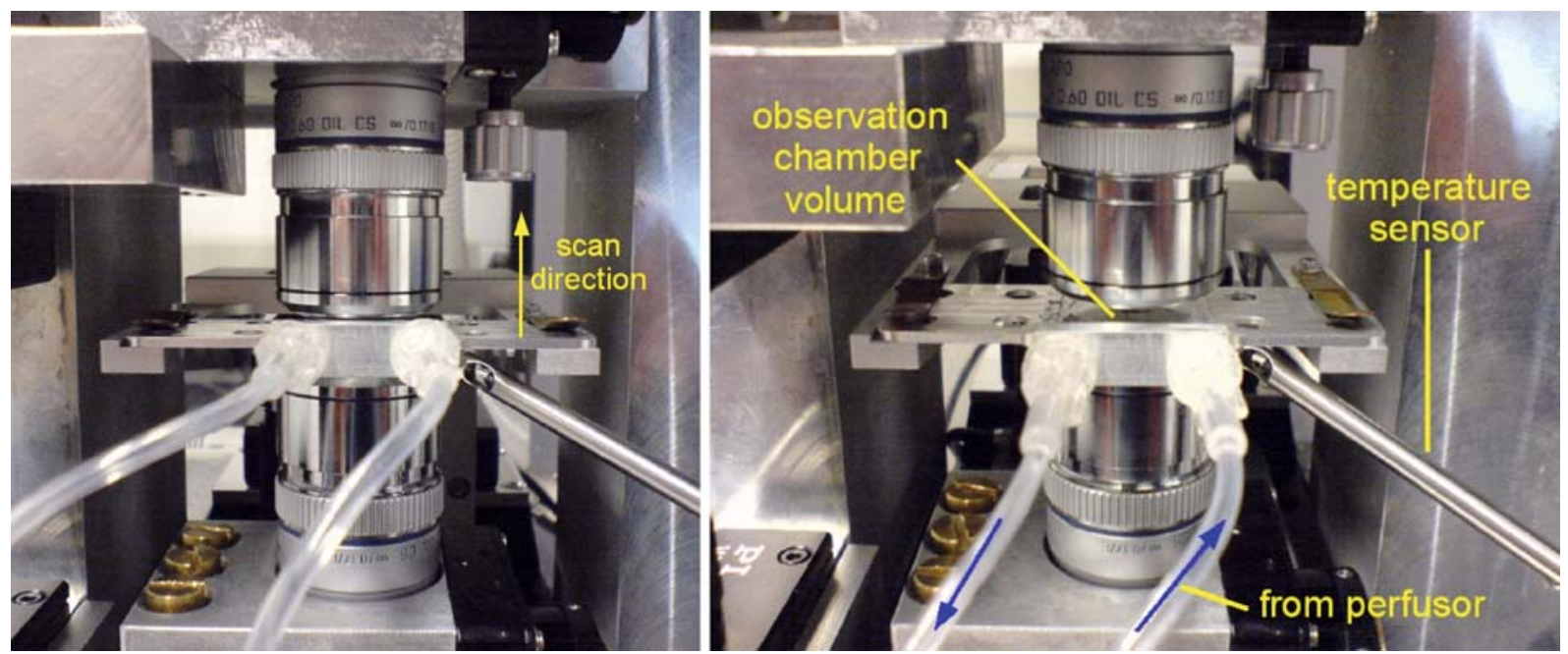

Figure 6. Photographs of the Vertico-SMI observation chamber positioned in the object space between the two opposite objective lenses. Via two connectors to flow-control systems, (culture) medium can be pumped in/out of the chamber to keep the specimens alive (blue arrows in the figure at the right). 
$37^{\circ} \mathrm{C}$, the whole system has to be heated for at least 2 hours. For automated Vertico-SMI in vivo procedures, the heating control unit can additionally be addressed via a serial interface. Additionally, we designed observation chambers with ordinary lateral object slide dimensions $(76 \mathrm{~mm} \times 26 \mathrm{~mm}$; length $\times$ width) and a height of $1.5 \mathrm{~mm}$ to increase the usable volume (up to $200 \mu \mathrm{l}$ ) for (culture) medium (see Figure 6). Via two connectors to flow-control systems, the observation chamber enables the in vivo specimens to be supplied with nutrients and $\mathrm{CO}_{2}$ and facilitates removal of waste products. The observation chamber consists of aluminium and is sealable from the top and bottom with ordinary $18 \mathrm{~mm} \times 18 \mathrm{~mm}$, $20 \mathrm{~mm} \times 20 \mathrm{~mm}$ or $22 \mathrm{~mm} \times 22 \mathrm{~mm}^{2}$ coverslips for three different models with appropriately decreased/ increased volume. The observation volume itself features a circular geometry to guarantee optimal inflow and outflow of the medium. As flow-control unit we are currently using a standard perfusor (Braun, Melsungen, Germany) for clinical applications, with a fluid reservoir up to $50 \mathrm{ml}$. The infusion speed can be regulated arbitrarily between $0.1 \mathrm{ml}$ and $99 \mathrm{ml}$ per hour.

\section{Optional Vertico-SMI set-up for localization microscopy}

In addition to size measurements of macromolecular complexes (in this report, nuclear nanostructures) in the range down to a few tens of nanometers as described above, SMI microscopy offers interesting perspectives in localization microscopy approaches to increase topological and effective optical resolution. Spectral precision distance microscopy / spectral position determination microscopy (SPDM) (Cremer et al. 1996, Bornfleth et al. 1998, van Oijen et al. 1998, Geisler et al. 2007) is a far-field lightmicroscopy approach based on labelling of neighbouring 'point-like' objects with different spectral signatures ('colours'; in general any photophysical property which can be used for optically discriminated registration including the time domain), spectrally selective registration (i.e. any mode of optically discriminated registration) and high precision position monitoring, i.e. a method of 'spectrally assigned' localization microscopy (SALM). In its original description (Cremer et al. 1996, 1999, Esa et al. 2000) it allowed the labelling of objects with any spectral signature, provided that in a given observation volume discrimination of the objects due to their spectral signature was achieved. Towards this goal, stochastic labelling schemes, time-resolved registration and sequential illumination were considered (Cremer et al. 2007). Experimentally, combined with careful calibration of optical aberrations and crosstalk, this allowed the measurement of positions and mutual distances between the 'point-like' fluorescent objects in a range far below the 'Abbe limit' of distance resolution in terms of the 'Sparrow limit' for objects of the same spectral signature. Examples of the application of SPDM in nuclear genome structure research include the analyses of the $\mathrm{BCR}-\mathrm{ABL}$ region correlated with chronic myeloid leukaemia (Esa et al. 2000); of conformational differences in the 3D-nanostructure of the immunoglobulin heavy-chain locus, a hotspot of chromosomal translocations in B lymphocytes (Esa et al. 2001); of the distribution of genes in the active and inactive X-chromosome territory (Dietzel et al. 1999); or of the nanostructure of imprinting domains in human cell nuclei (Rauch et al. 2008).

SPDM was also applied to measure localizations of single fluorochrome molecules and the distance between them in a range $>10 \mathrm{~nm}$ (Heilemann et al. 2002, 2004), i.e. in a range too large for FRET techniques but considerably below the optical resolution of conventional, confocal, or 4Pi microscopy. The SPDM principle requires, however, that in a given observation volume (defined, for example, by the full width at half maximum (FWHM) of the PSF of the microscope system used), there is just one object with a given spectral signature to be measured at a given time. If photostable spectral signatures are to be used for simultaneous registration, this means that, for example, the nanostructure of membrane rafts with many (e.g. hundreds) closely adjacent proteins of the same type cannot be resolved, because the reasonable number of useful different spectral signatures is still limited (presently $\sim 7$ ). However, a macromolecular effective optical resolution has become possible by a further development of localization microscopy using different spectral signatures, such as types of blinking statistics (Lidke et al. 2005) and especially photoactivated localization microscopy (PALM/FPALM). This substantial progress in laser-optical nanoscopy was recently introduced by the groups of E. Betzig and S. Hess (Betzig et al. 2006, Hess et al. 2006, 2007). Similar methods were simultaneously described, such as 

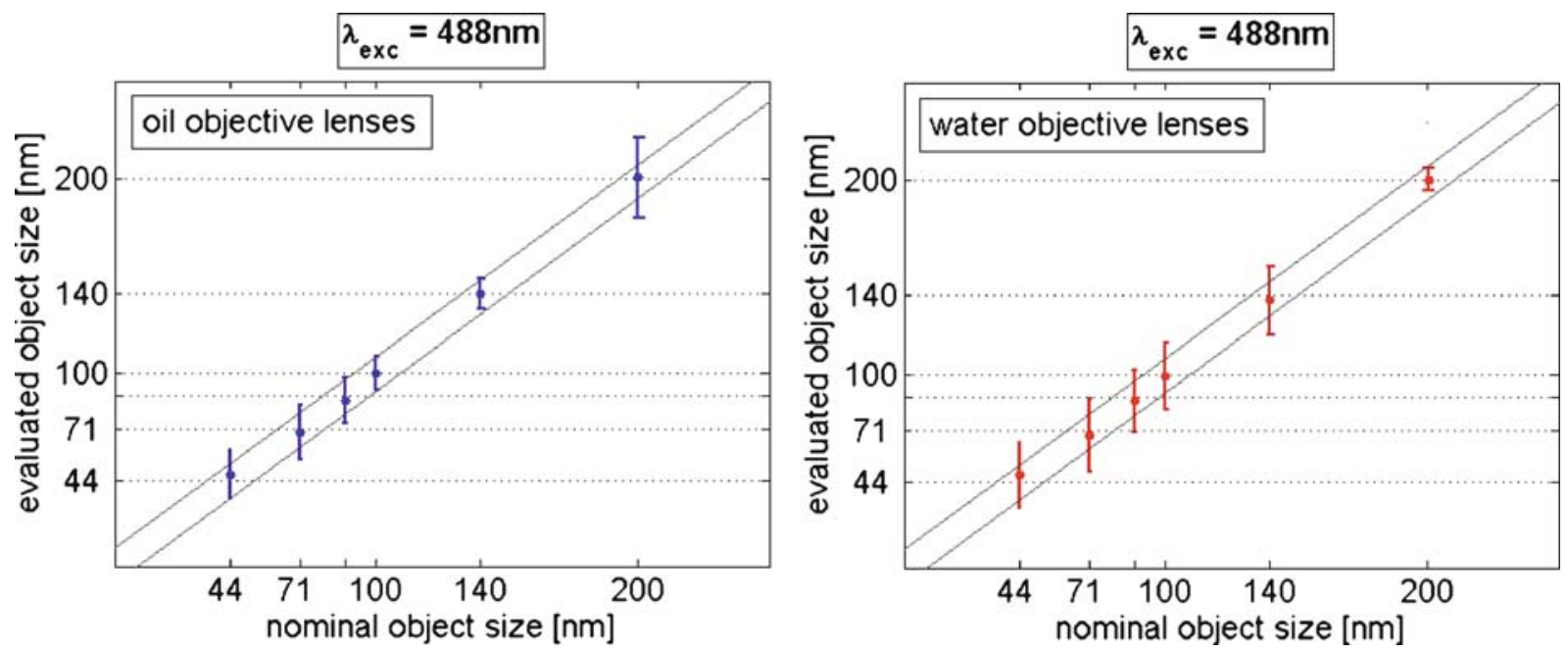

Figure 7. Evaluated object sizes (mean and standard deviation, indicated by bars) plotted against the nominal size of 44-200 nm beads using an excitation wavelength of $\lambda_{\mathrm{exc}}=488 \mathrm{~nm}$ for oil- (left) and water- (right) immersion objective lenses. The two lines indicate a $\pm 10 \mathrm{~nm}$ error interval.

STORM (sub-diffraction-limited imaging by stochastic optical reconstruction) (Rust et al. 2006) or PALMIRA (PALM with independently running acquisition) (Geisler et al. 2007). In the same way as in the SPDM method realized so far, these approaches are all based on very precise (nanometer) positioning of sub-wavelength-sized objects, in this case single molecules, detecting just one object of a given spectral signature in the observation volume at a given time. However, whereas the SPDM method so far had used objects labelled with different photostable spectral signatures to resolve them spatially within the observation volume given by the microscope PSF used, (F)PALM/STORM/PALMIRA techniques presently allow an effective (lateral) optical resolution down to $10-20 \mathrm{~nm}$ even when all objects are labelled with the same type of fluorochrome. This became possible by the use of fluorochromes with photoconvertible spectral signatures instead of photostable ones. These methods are currently in
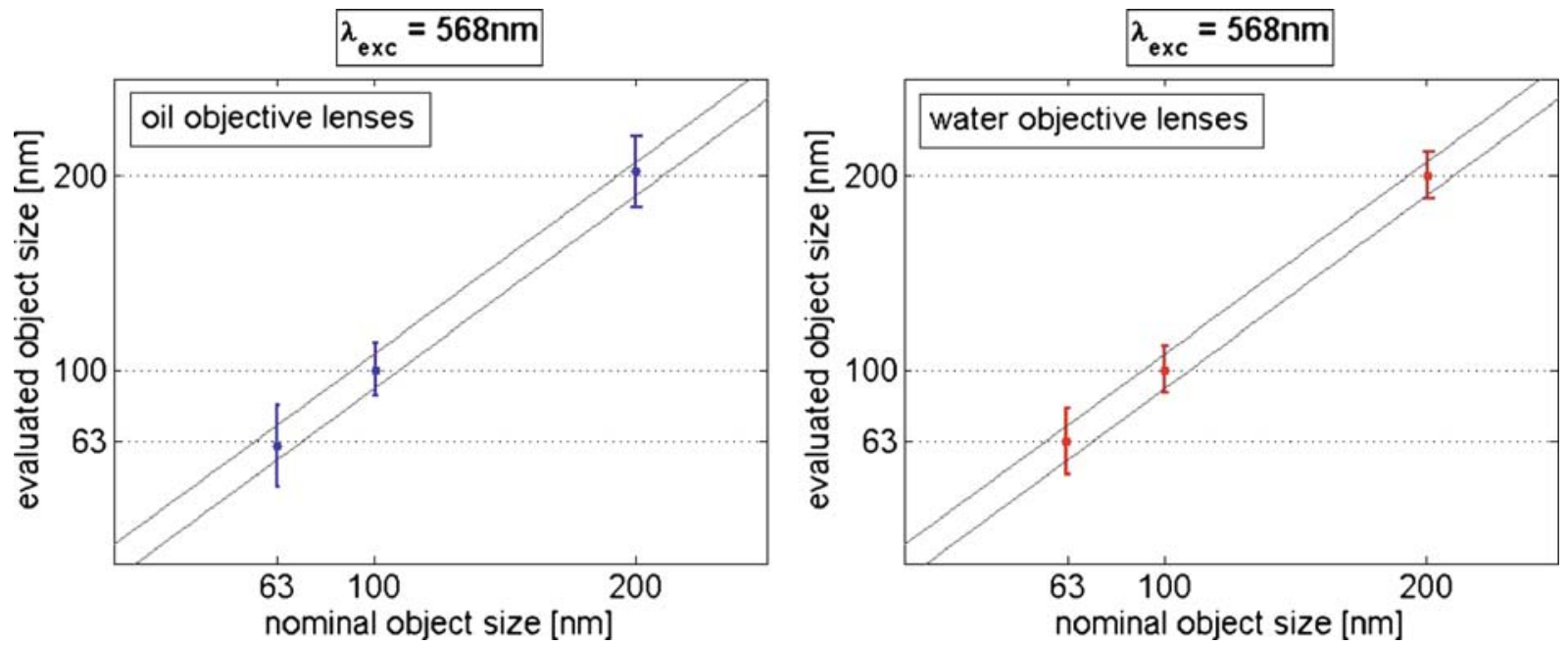

Figure 8. Evaluated object sizes (mean and standard deviation, indicated by bars) plotted against the nominal size of 63-200 nm beads using an excitation wavelength of $\lambda_{\mathrm{exc}}=568 \mathrm{~nm}$ for oil- (left) and water- (right) immersion objective lenses. The two lines indicate a $\pm 10 \mathrm{~nm}$ error interval. 

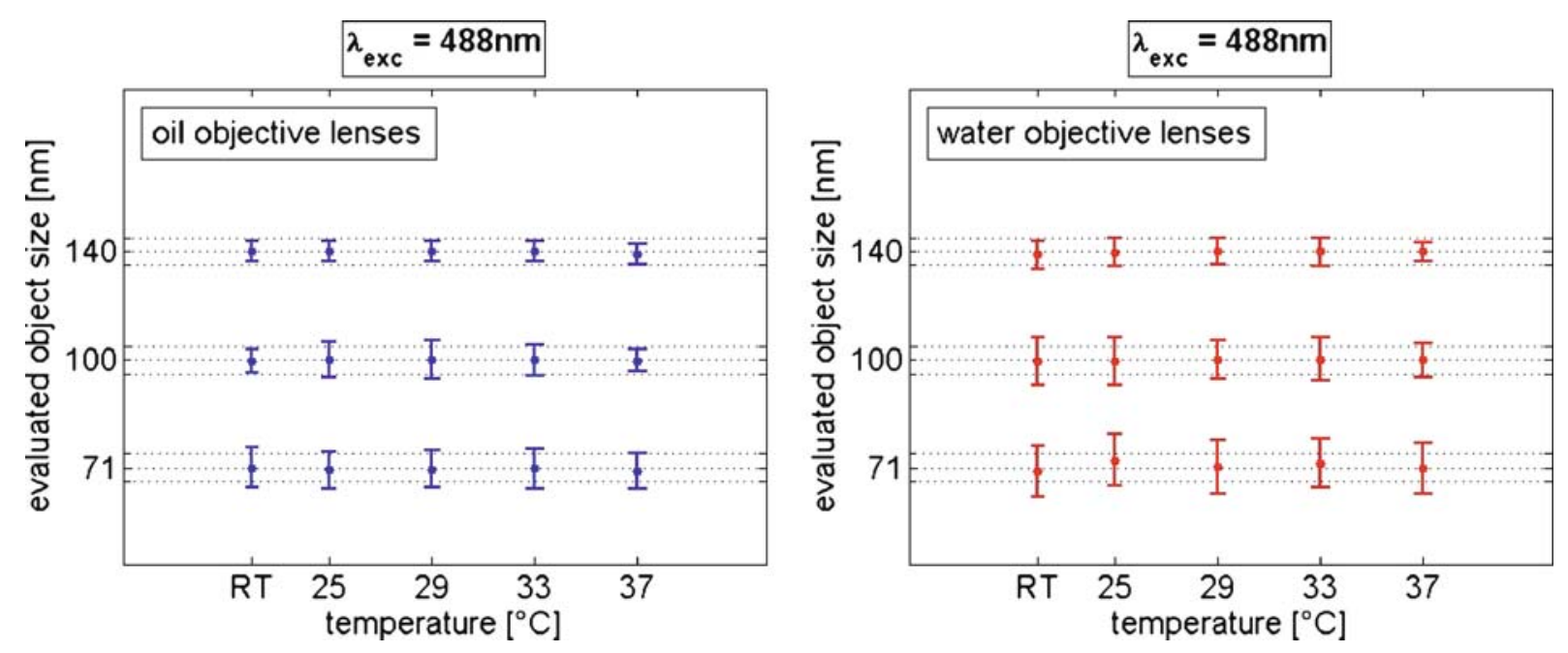

Figure 9. Evaluated object sizes (mean and standard deviation, indicated by bars) with data acquired at different temperatures using oil- (left) and water- (right) immersion objective lenses with $\lambda_{\text {exc }}=488 \mathrm{~nm}$. For room temperature (RT) and for $T=25^{\circ} \mathrm{C}, 29^{\circ} \mathrm{C}, 33^{\circ} \mathrm{C}$ and $37^{\circ} \mathrm{C}$, beads with diameters of $71 \mathrm{~nm}, 100 \mathrm{~nm}$ and $140 \mathrm{~nm}$ were evaluated, resulting in no significant differences. For comparison, a $\pm 10 \mathrm{~nm}$ interval is indicated for each bead size by two (dashed) lines.

rapid further development (Geisler et al. 2007), at present allowing the resolution of the $3 \mathrm{D}$ morphology of nanoscopic cellular structures at a resolution of 50-60 nm (Huang et al. 2008).

For use of the Vertico-SMI set-up for highprecision localization microscopy, an optional optical configuration was implemented allowing easy switching between the SMI and two-dimensional (2D) localization microscopy modes. For the 'localization mode', the interferometric adjustment was removed by blocking the lower part of the laser beam in the interferometer. The laser beam in the object space was no longer collimated (as needed for the 'SMI mode') but was appropriately focused using an additional focusing lens for the (upper) objective lens.

\section{Results}

\section{Characteristics of the system}

We analysed the fundamental system properties by accurate characterization studies using fluorescent microspheres of known size (beads) under standard conditions, i.e. at room temperature (RT: $22^{\circ} \mathrm{C}$ ), to test the overall system behaviour. In addition, we controlled the system at different temperatures and the results were compared with those obtained at room temperature. The results shown here were evaluated using the data analysis tool described earlier, i.e. the automatic object identification and fitting procedures, thus providing reliability with the help of a standardized data evaluation procedure.

\section{System properties under standard conditions}

Calibration runs were performed at room temperature using oil- and water-immersion objective lenses. For this, green and yellow beads (Duke Scientific Corporation, and Molecular Probes (Invitrogen), Germany) for both (currently installed) laser sources, with diameters of $44 \mathrm{~nm}$ to $200 \mathrm{~nm}$ ('nominal object sizes') and the manufacturer's stated uncertainty of $5-15 \%$, were distributed homogeneously and prepared on conventional coverslips (thickness $\sim 155-160 \mu \mathrm{m})$, mounted in embedding medium (glycerol, refractive index $n=1.440$ ) and fixed on a standard object slide.

Figures 7 and 8 depict the results of bead measurements with diameters of $44 \mathrm{~nm}, 71 \mathrm{~nm}, 88 \mathrm{~nm}, 100 \mathrm{~nm}$, $140 \mathrm{~nm}$ and $200 \mathrm{~nm}$ (excitation at $\lambda_{\text {exc }}=488 \mathrm{~nm}$ ) and $63 \mathrm{~nm}, 100 \mathrm{~nm}$ and $200 \mathrm{~nm}$ (excitation at $\lambda_{\text {exc }}=568 \mathrm{~nm}$ ) spanning the range in which the SMI can reliably evaluate object sizes. The evaluated mean object sizes with Standard Deviations (SD) (500-2500 objects for each bead size) are plotted against the nominal object sizes as given by the manufacturer. For a better visualization two lines are drawn which 

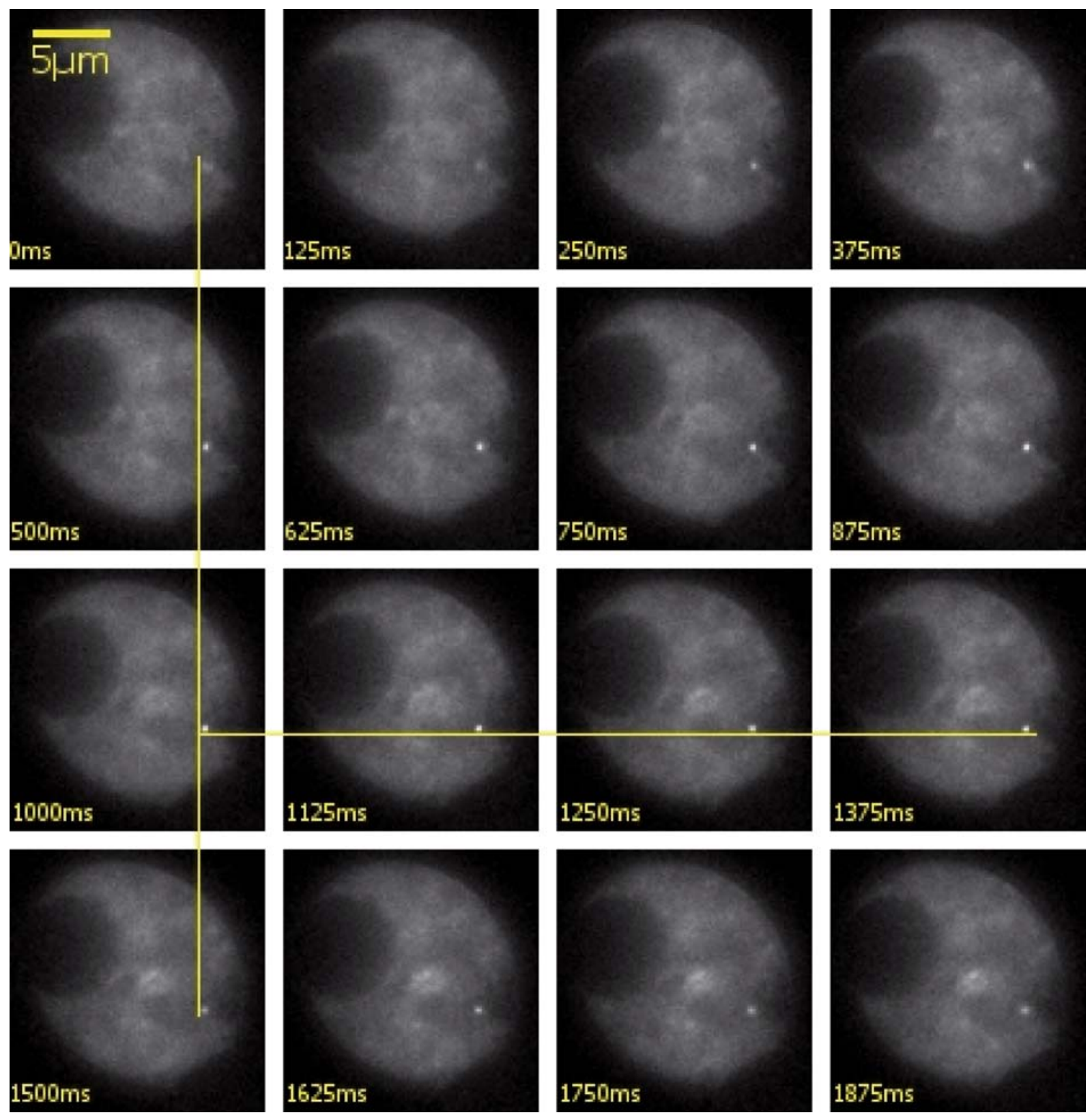

Figure 10. Image sequence of a live U2OS cell during the sampling procedure (passing through the focus) with an axial step size of $40 \mathrm{~nm}$. The images show a tet-operator repeat locus integrated into a telomere region during the interphase of the cell cycle. The time interval between successive frames was about $125 \mathrm{~ms}$; the axial step size was $40 \mathrm{~nm}$, resulting in an overall DAQ-time of about 2 seconds for the entire 3D data stack. The movement of the object was negligible during this time period.

indicate a $\pm 10 \mathrm{~nm}$ error interval. As the results show, the SDs for the objects acquired with water objective lenses were slightly larger than those obtained with oil objective lenses, except for the evaluated objects of $200 \mathrm{~nm}$ beads. This may be ascribed to a slight refractive index dependence of the SMI-sensitive range for object size evaluation.

In order to improve the system properties for livecell imaging, a rapid DAQ is essential to minimize the effects of object movement on the AID. Assum- 
ing a reasonable fluorescence signal, resulting in an appropriate SNR, we reduced the time for acquiring a complete 3D SMI data stack down to $1.8 \mathrm{~s}$ (about 15 frames/s).

\section{System properties under the influence of elevated} temperature

For the improvement of long-term in vivo measurements, we tested the system properties in order to guarantee the stability of the interferometer and the object sampling procedure. Figure 9 depicts the results of measurements using beads with diameters of $71 \mathrm{~nm}, 100 \mathrm{~nm}$ and $140 \mathrm{~nm}$ at room temperature and at $25^{\circ} \mathrm{C}, 29^{\circ} \mathrm{C}, 33^{\circ} \mathrm{C}$ and $37^{\circ} \mathrm{C}$ for both oil- and water-immersion objective lenses at an excitation wavelength of $\lambda_{\mathrm{exc}}=488 \mathrm{~nm}$. An error interval of $\pm 10 \mathrm{~nm}$ is indicated by the two lines for each bead diameter. After changing the temperature level, the microscope was left to rest for at least 2 hours before the sampling procedure was started, thus allowing the microscope system to adapt to the respective environmental conditions. Compared with room temperature (RT), no significant differences in evaluated object sizes at higher temperatures were found, indicating the stability of the Vertico-SMI.

From this, we conclude that using a reasonable experimental set-up, in particular the establishment of thermal equilibrium between the environment, the basic microscope set-up, the individual optical components and the object positioning units, any additional uncertainty in size measurements caused by thermal effects can be made negligibly small. This is important for in vivo applications since no further parameters need be considered during live-cell measurements and analysis. Consequently, additional effects on the SMI measurements during in vivo procedures result only from the object's mobility.

\section{Live-cell imaging of nuclear structures}

The Vertico-SMI was applied to studies of living biological specimens using human osteosarcoma (U2OS) cells with tet-operator repeats stably integrated into chromosome telomeres. These cells represent a well-characterized system for which extension and mobility patterns of the individual loci were already well known, making it an ideal candidate for our Vertico-SMI studies. The tet-inserts were labelled with tet-repressor fused to the auto- fluorescent GFP domain and the cells were grown on a standard $18 \mathrm{~mm} \times 18 \mathrm{~mm}$ coverslip. This coverslip was then mounted into the observation chamber with nail polish; cell viability was maintained by pumping Hepes culture medium into the observation volume via the perfusor unit as described previously (flow rate $\sim 30 \mathrm{ml} / \mathrm{h}$ ).

Subsequently, the observation chamber was positioned in the object space between the two opposite objective lenses. For the results shown here, an excitation wavelength of $\lambda_{\text {exc }}=488 \mathrm{~nm}$ was used and the incubator chamber of the Vertico-SMI microscope was operated at $37^{\circ} \mathrm{C}$. The SNR of most of the loci was good enough to reduce data acquisition times down to 2 seconds for a complete 3D SMI data stack. As a consequence, the influence of object mobility on the acquired intensity profiles was found to be negligible. Figure 10 depicts an image sequence of the raw data of a tet-operator locus in a live U2OS cell during the sampling procedure, i.e. passing through the focus with an axial step size of $40 \mathrm{~nm}$ (maximum intensity projection). The corresponding AID of the object is shown in Figure 11 .

In summary, the results demonstrate that for the case studied here the influence of object mobility on the SMI AID can be neglected, as data acquisition is fast in comparison to the object mobility. These data show the applicability of the SMI principle in terms

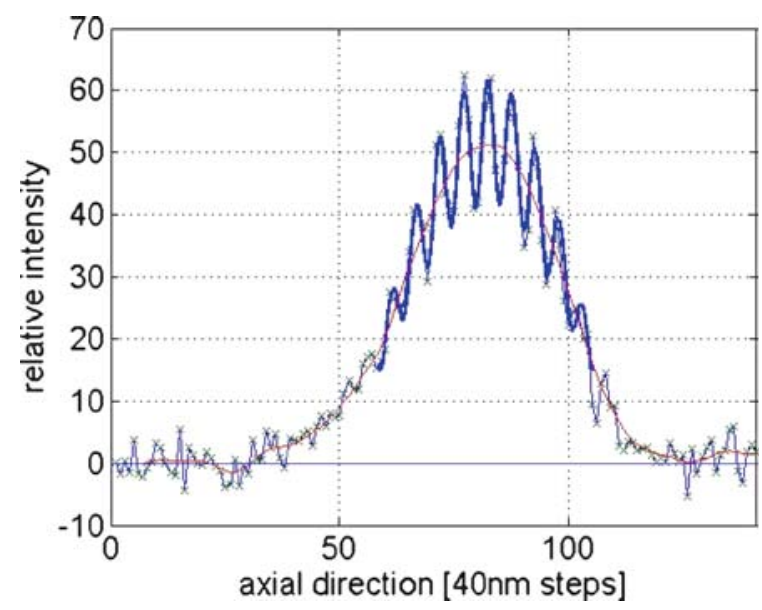

Figure 11. In vivo signature of a tet-repressor GFP locus measured under live cell conditions. Owing to the rapid 3D SMI imaging procedure, the influences of movement of the object on the AID were negligible. 

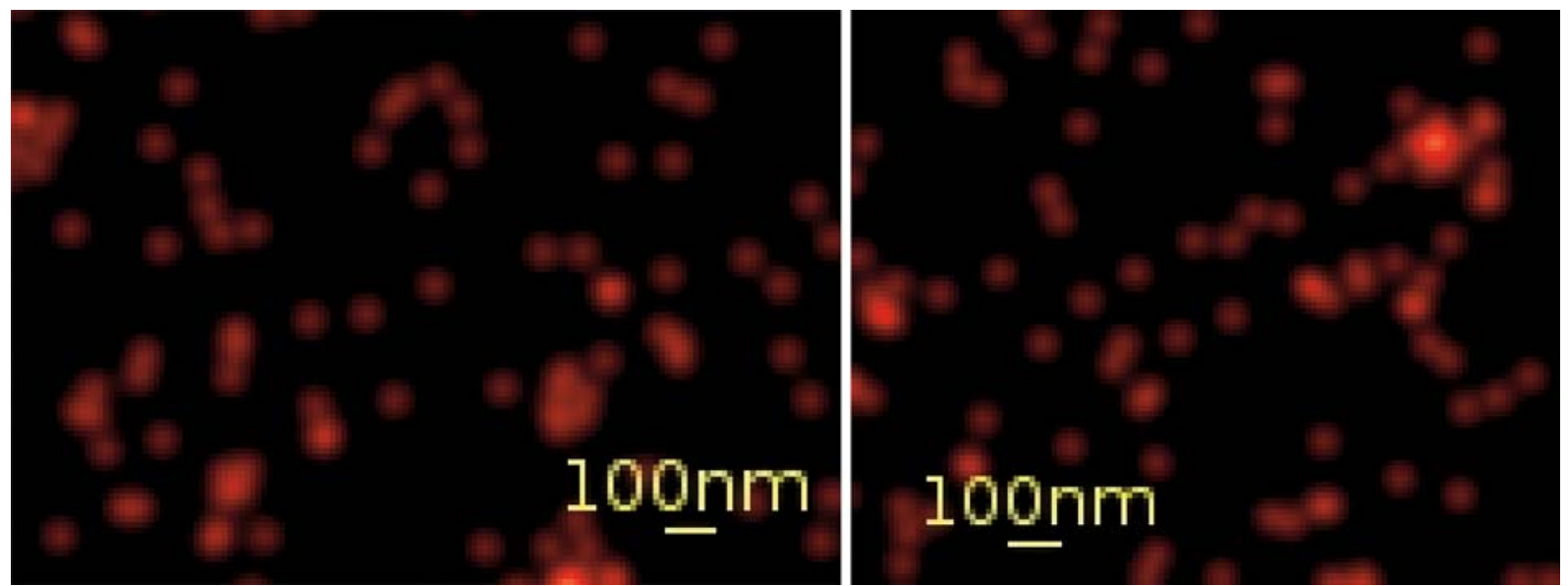

Figure 12. Positions $(x, y)$ of nuclear pore proteins(p62) in a human MCF7 cell determined with the Vertico-SMI 'localization mode'. The left and right image show two different example areas from p62 protein distribution on the nuclear membrane of the same cell.

of determining structural characteristics even using living specimens.

\section{Localization microscopy mode}

With the optional optical configuration described above (Vertico-SMI 'localization mode') we have successfully performed 2D localization microscopy (object plane) of various cellular proteins.

Here we present a technical example: In this preliminary approach a nuclear pore protein (p62) was immunolabelled using an Alexa488-coupled secondary antibody. The sample was then photobleached to the single-molecule level, allowing the single-molecule localization performance of the SMI within a typical biological specimen to be tested. Employing photo-blinking (Lidke 2005) of the remaining 'physically modified' fluorophores does of course not reach the sensitivity and hence image quality possible when using true photoactive fluorophores. Nevertheless, it permitted us to independently localize a limited number of fluorophores within one observation volume, and thereby improve imaging performance. Figure 12 shows two reconstructed excerpts $(\sim 1.2 \mu \mathrm{m} \times 0.8 \mu \mathrm{m}$ (left); $\sim 1.2 \mu \mathrm{m} \times 1.0 \mu \mathrm{m}$ (right) (width $\times$ height) $)$ from a larger acquisition (total field of view $\sim 10 \mu \mathrm{m} \times$ $6.5 \mu \mathrm{m})$. The whole raw data stack contained 4816 discrete points which were evaluated by a special program in which a 2D Gaussian was fitted to obtain the position estimates.
These Gaussians were fitted using the LevenbergMarquardt algorithm to solve the weighted leastsquares problem, with weights chosen to reflect the combination of Gaussian read-out and Poisson photon noise. This method delivers estimates of the error in the fitted parameters (by analysing the gradient at completion); a mean localization accuracy of about $15 \mathrm{~nm}$ was estimated.

\section{Discussion}

In previous studies, spatially modulated illumination microscopy has shown its applicability to the analysis of biological objects in regions far below the limits of fluorescence light microscopy, which are about $200 \mathrm{~nm}$ laterally and $450 \mathrm{~nm}$ axially for confocal laser scanning microscopy. Up to now, only fixed cells have been analysed using the first prototype of the SMI microscope. Here, we have presented some applications using a newly developed upright configuration (Vertico-SMI microscope) allowing (long-term) in vivo analysis of biological samples and providing all the capabilities of the SMI. The Vertico-SMI set-up represents a compact and stable vertical arrangement of an SMI microscope; the results show that the system is working stably and with the required accuracy, including when using water-immersion objective lenses.

The size determination of measured fluorescent microspheres with diameters ranging from $44 \mathrm{~nm}$ to 
$200 \mathrm{~nm}$ for oil- and water-immersion objective lenses provided reliable results under standard conditions (room temperature). With different test configurations, i.e. hardware specifications, stability of the interferometer and very accurate calibration procedures for different temperature levels, it also was shown that even under the influence of heat (e.g. normal body temperature) the microscope provides trustworthy data. For live-cell measurements the system has to fulfil several criteria, of which two in particular were described in order to show the overall potential of the Vertico-SMI microscope. First, very small objects (beads with nominal diameters down to $44 \mathrm{~nm}$ ) have successfully been measured. The second criterion concerns data acquisition during in vivo procedures; very precise object sampling is essential for live-cell measurements and vigorous effort was directed to achieving very high DAQ rates. In this context, we succeeded in reducing the time for acquiring a complete 3D SMI data stack down to currently $1.8 \mathrm{~s}$ under the assumption of a reasonable SNR. Concerning size determination, this is important with respect to object mobility for minimizing disturbing effects on the object's axial intensity distribution.

We verified the above exemplified results with the measurement and analysis of living biological specimens, acquiring reliable intensity profiles of the tetoperator loci under in vivo conditions (U2OS cells). The results obtained indicate that the Vertico-SMI microscope can now be considered a fully-fledged SMI microscope system able to address multiple biological questions to elucidate the fundamental principles of subnuclear complexes under live-cell conditions. The next step will be a more detailed analysis of intranuclear mobility patterns and the effects of standard fixation procedures at the overall genome structure and subnuclear levels.

Owing to the structured illumination in the axial direction it is possible to discriminate very precisely mobility patterns of individual objects in at least one dimension. This can be achieved by leaving the objects to be observed stationary in the focal plane of the objective lens and tracking the objects' trajectories in the standing-wave field instead of sampling it (Lanni et al. 1986). With such a procedure, diffusion parameters of small objects might be accessible with a precision in the nanometer range.

The Vertico-SMI allows easy switching between two optical configurations ('SMI' and 2D 'localization mode'). To give a technical example for the latter application, in preliminary experiments we successfully performed localization microscopy, using the principles of SPDM combined with the use of a 'physically modifiable' fluorophore. The estimated localization accuracy of $\sim 15 \mathrm{~nm}$ for 4816 discrete points determined in the cell used for the nanoimaging in Figure 12 indicates the applicability of the system for localization microscopy measurements. To make full use of this potential, fluorochrome labelling, registration conditions and evaluation algorithms have to be further improved and tested. It may be noted that in these preliminary localization microscopy images, the p62 (and hence the nuclear pore complex (NPC)) distribution appeared not to be homogeneous but in many cases non-random, indicating a connection of NPCs with adjacent (cytoplasmic or nuclear) structures. This phenomenon had already been suggested by $4 \mathrm{Pi}$ microscopy images of the NPC distribution of such cells (K. Maecken, A. Naber, C. Cremer et al., unpublished information); owing to its superior effective optical resolution, localization microscopy, however, allows a much more detailed analysis.

So far, the Vertico-SMI has been shown to allow localization microscopy in the lateral $(x, y)$ dimensions (object plane). There are several possibilities for realizing high-resolution localization microscopy in three dimensions; for example, it is possible to use 2-photon excitation in order to facilitate optical sectioning in thick transparent specimens. In combination with structured illumination for detection (SMI) this will greatly improve the possibilities of high-resolution microscopy. Another possibility would be to perform 3D position determination SMI microscopy in the 1-photon excitation mode. Numerical simulations (Albrecht et al. 2001) have indicated that structured illumination approaches such as SMI microscopy are suitable to achieve this goal, even assuming relatively low numbers of fluorescence photons detected.

To summarize, all of the Vertico-SMI system properties originally claimed for SMI live-cell procedures (compact and stable (optical) configuration, high positioning accuracy and stability of the object positioning/sampling devices as well as automated and rapid data acquisition routines) point to the potential of the SMI microscope set-up for fast high-resolution imaging.

In addition, it is anticipated that the combination of SALM methods (SPDM/PALM/FPALM/ 
PALMIRA/STORM) with novel developments in optical nanoscopy-such as the SMI approach reported here, stimulated emission depletion (STED) microscopy (Donnert et al. 2006), saturated patterned excitation microscopy (Heintzmann et al. 2002, Gustafsson 2005), or other forms of structured illumination -will allow bridging of the gap in resolution between ultrastructure methods (nanometer resolution) and visible light far-field microscopy (conventionally hundreds of nanometer resolution). This will allow it to image the same cellular structure both ultrastructurally and light-microscopically at almost similar (down to molecular) resolution, allowing truly correlative microscopy of cellular nanostructures.

\section{Acknowledgements}

We thank Kathrin Maecken and PD Dr Andreas Naber from the University of Karlsruhe for the biological specimens used for preliminary 2D localization microscopy approaches. We would also like to thank Prof. Dr Markus Sauer for supervision of the $\mathrm{PhD}$ thesis of one of the authors (J.R.). We gratefully acknowledge financial support within the Molecular Imaging Sixth European Framework Program 'Integrated Technologies for In-Vivo Molecular Imaging', Project no. LSHG-CT-2003-503259 and the DFG priority program 1128 'Optical analysis of the structure and dynamics of supra-molecular biological complexes'. Dr. Udo Birk gratefully acknowledges support through the EU project MEIF-CT2006-041827. Special thanks are given to the fine mechanics workshop of the Kirchhoff Institute of Physics for providing a number of critical components for the Vertico-SMI set-up.

\section{References}

Albrecht B, Failla AV, Heintzmann R, Cremer C (2001) Spatially modulated illumination microscopy: Online visualization of intensity distribution and prediction of nanometer precision of axial distance measurements by computer simulations. J Biomed Opt 6: 292-299.

Albrecht B, Failla AV, Schweitzer A, Cremer C (2002) Spatially modulated illumination microscopy allows axial distance resolution in the nanometer range. Appl Opt 41: 80-87.

Baddeley D, Batram C, Weiland Y, Cremer C, Birk UJ (2007) Nanostructure analysis using spatially modulated illumination microscopy. Nat Protoc 2: 2640-2646.
Bailey B, Farkas DL, Taylor DL, Lanni F (1993) Enhancement of axial resolution in fluorescence microscopy by standing-wave excitation. Nature 366: 44-48.

Betzig E, Patterson GH, Sougrat R et al. (2006) Imaging intracellular fluorescent proteins at nanometer resolution. Science 313(5793): 1642.

Bornfleth H, Sätzler K, Eils R, Cremer C (1998) High-precision distance measurements and volume-conserving segmentation of objects near and below the resolution limit in three-dimensional confocal fluorescence microscopy. J Microsc 189(2): 118-136.

Cremer C, Hausmann M, Bradl J, Rinke B (1996) Verfahren zur multispektralen Präzisionsdistanzmessung in biologischen Mikroobjekten (Procedure for multispectral precision distance measurements in biological microobjects). German Patent application No. 196.54. 824.1/DE, submitted Dec 23, 1996. European Patent EP 1997953660, 08.04.1999; Japanese Patent JP 1998528237, 23.06.1999; United States Patent US 09331644, 25.08.1999.

Cremer C, Edelmann P, Bornfleth H et al. (1999) Principles of spectral precision distance confocal microscopy for the analysis of molecular nuclear structure. In: Jähne B, Haußecker $\mathrm{H}$, Geißler P, eds. Handbook of Computer Vision and Applications. San Diego: Academic Press 3(41): 839-885.

Cremer C, Failla AV, Albrecht B (2007) Far field light microscopical method, system and computer program product for analyzing at least one object having a subwavelength size. US Patent 7,298,461 B2, filed Oct 9, 2001.

Dietzel S, Schiebel K, Little G et al. (1999) The 3D positioning of ANT2 and ANT3 genes within female X chromosome territories correlates with gene activity. Exp Cell Res 252(2): 363-375.

Donnert G, Keller J, Medda R et al. (2006) Macromolecular-scale resolution in biological fluorescence microscopy. Proc Natl Acad Sci U S A 103(31): 11440.

Dyba M, Hell S (2002) Focal spots of size $\lambda / 23$ open up far-field fluorescence microscopy at $33 \mathrm{~nm}$ axial resolution. Phys Rev Lett 88(16): 163901.

Egner A, Jakobs S, Hell S (2002) Fast 100 nm resolution threedimensional microscope reveals structural plasticity of mitochondria in live yeast. Proc Natl Acad Sci U S A 99(6): 3370-3375.

Esa A, Edelmann P, Kreth G et al. (2000) Three-dimensional spectral precision distance microscopy of chromatin nanostructures after triple-colour DNA labelling: a study of the BCR region on chromosome 22 and the Philadelphia chromosome. J Microsc 199(2): 96-105.

Esa A, Coleman AE, Edelmann P, Silva S, Cremer C, Janz S (2001) Conformational differences in the 3-D nanostructure of the immunoglobulin heavy-chain locus, a hotspot of chromosomal translocations in B lymphocytes. Cancer Genet Cytogenet 127(2): 168-173.

Failla A, Cavallo A, Cremer C (2002a) Subwavelength size determination by spatially modulated illumination virtual microscopy. Appl Opt 41: 6651-6659.

Failla AV, Spöri U, Albrecht B, Kroll A, Cremer C (2002b) Nanosizing of fluorescent objects by spatially modulated illumination microscopy. Appl Opt 41(34): 7275-7283.

Failla AV, Albrecht B, Spöri U et al. (2003) Nanostructure analysis using spatially modulated illumination microscopy. ComPlexUs 1: 77-88.

Frohn J, Knapp H, Stemmer A (2000) True optical resolution beyond the Rayleigh limit achieved by standing wave illumination. Proc Natl Acad Sci U S A 97(13): 7232-7236. 
Frohn J, Knapp H, Stemmer A (2001) Three-dimensional resolution enhancement in fluorescence microscopy by harmonic excitation. Opt Lett 26(11): 828-830.

Geisler C et al. (2007) Resolution of $\lambda / 10$ in fluorescence microscopy using fast single molecule photo-switching. Appl Phys A: Mater Sci Process 88(2): 223-226.

Gustafsson M (2005) Nonlinear structured-illumination microscopy: wide-field fluorescence imaging with theoretically unlimited resolution. Proc Natl Acad Sci U S A 102(37): 13081-13086.

Gustafsson M, Agard D, Sedat J (1995) Sevenfold improvement of axial resolution in 3D widefield microscopy using two objective lenses. Proc SPIE 2412: 147-156.

Gustafsson M, Agard D, Sedat J (1996) 3D widefield microscopy with two objective lenses: experimental verification of improved axial resolution. Proc SPIE 2655: 62-65.

Heilemann M, Herten DP, Heintzmann R et al. (2002) Highresolution colocalization of single dye molecules by fluorescence lifetime imaging microscopy. Anal Chem 74(14): 3511-3517.

Heilemann M, Tinnefeld P, Sanchez Mosteiro G, Garcia Parajo M, Van Hulst NF, Sauer M (2004) Multistep energy transfer in single molecular photonic wires. J Am Chem Soc 126(21): 6514-6515.

Heintzmann R, Jovin T, Cremer C (2002) Saturated patterned excitation microscopy - aconcept for optical resolution improvement. J Opt Soc Am A 19(8): 1599-1609.

Hell S, Lindek S, Cremer C, Stelzer E (1994) Measurement of 4piconfocal point spread function proves $75 \mathrm{~nm}$ axial resolution. Appl Phys Lett 64(11): 1335-1337.

Hendzel M, Bazett-Jones D (1997) Fixation-dependent organization of core histones following DNA fluorescent in situ hybridization. Chromosoma 106(2): 114-123.

Hess ST, Girirajan T, Mason M (2006) Ultra-high resolution imaging by fluorescence photoactivation localization microscopy. Biophys $J$ 91(11): 4258.

Hess ST, Gould TJ, Gudheti MV, Maas SA, Mills KD, Zimmerberg J (2007) Dynamic clustered distribution of hemagglutinin resolved at $40 \mathrm{~nm}$ in living cell membranes discriminates between raft theories. Proc Natl Acad Sci U S A 104(44): 17370-17375.
Hildenbrand G, Rapp A, Spöri U, Wagner C, Cremer C, Hausmann M (2005) Nano-sizing of specific gene domains in intact human cell nuclei by spatially modulated illumination light microscopy. Biophys J 88(6): 4312-4318.

Huang B, Wang W, Bates M, Zhuang X (2008) Three-dimension super-resolution imaging by stochastic optical reconstruction microscopy. Science 319(5864): 810-813.

Klar T, Jakobs S, Dyba M, Egner A, Hell S (2000) Fluorescence microscopy with diffraction resolution barrier broken by stimulated emission. Proc Natl Acad Sci U S A 97: 8206-8210.

Kozubek S, Lukasova E, Amrichova J, Kozubek M, Liskova A, Slotova J (2000) Influence of cell fixation on chromatin topography. Anal Biochem 282(1): 29-38.

Lanni F, Taylor DL, Waggoner AS (1986) Standing wave luminescence microscopy. US Patent 4,621,911.

Lidke AK, Rieger B, Jovin TM, Heintzmann R (2005) Superresolution by localization of quantum dots using blinking statistics. Optics Express 13(18): 7052-7062.

Martin S, Failla AV, Spöri U, Cremer C, Pombo A (2004) Measuring the size of biological nanostructures with spatially modulated illumination microscopy. Mol Biol Cell 15(5): 2449.

Mathée H, Baddeley D, Wotzlaw C, Fandrey J, Cremer C, Birk U (2006) Nanostructure of specific chromatin regions and nuclear complexes. Histochem Cell Biol 125(1):75-82.

Rauch J, Knoch TA, Solovei I et al. (2008) Lightoptical precision measurements of the active and inactive Prader-Willi syndrome imprinted regions in human cell nuclei. Differentiation 76(1): 66-83.

Rust M, Bates M, Zhuang X (2006) Sub-diffraction-limit imaging by stochastic optical reconstruction microscopy (STORM). Nat Methods 3: 793-796.

Spöri U, Failla A, Cremer C (2004) Superresolution size determination in fluorescence microscopy: a comparison between spatially modulated illumination and confocal laser scanning microscopy. J Appl Phys 95: 8436-8443.

van Oijen A, Köhler J, Schmidt J, Müller M, Brakenhoff GJ (1998) 3-Dimensional super-resolution by spectrally selective imaging. Chem Phys Lett 292: 183-187. 